\title{
Comparison of the recoil of conventional and electromagnetic cannon
}

\author{
Edward M. Schmidt \\ Army Research Laboratory, Aberdeen Proving \\ Ground, MD 21005-5066, USA
}

The recoil from an electromagnetic (EM) railgun is discussed and compared with that from conventional, propellant gas driven cannon. It is shown that, under similar launch conditions, the recoil of the EM gun is less than that of the powder gun; however, use of a muzzle brake on a powder gun can alter this relative behavior.

\section{Introduction}

Electromagnetic launchers have experienced renewed interest over the past ten to fifteen years. They have the potential to deliver both hypervelocity and hyperenergy launch while eliminating the potential hazards associated with gun propellants and reducing the size of the cartridge. The two main classes of electromagnetic launcher are the coil gun and the railgun. Within the latter, there are many configurations of rails such as simple, augmented, transaugmented, contraaugmented, etc. For the present paper, only simple railguns are considered, Fig. 1. These have two conducting rails separated by adjacent insulators that run the complete length of the launcher. The projectile is accelerated by the Lorentz Force developed from the interaction of the magnetic field (generated by current flowing up one rail and down the other) with the current passing through the armature. The Lorentz Force is expressed as $F_{L}=q v \times B$. In the railgun, the magnitude of this force is $F_{L}=L^{\prime} I^{2} / 2$, where $L^{\prime}$ is the inductance gradient of the rails (typical values are $0.35-0.55 \mu \mathrm{H} / \mathrm{m})$ and $I$ is the current in amperes. The current is provided by a suitable pulsed power system. This could be a capacitor bank, battery-inductor array, or a rotating machine such as an homopolar generator or compulsator. Typically, $L^{\prime}$ is a constant and $I$ varies with time depending upon the characteristics of the pulsed power system in use. Since there is current flowing in the rails which are immersed in the magnetic field of their partner, a rail repulsion force is developed which must be resisted by the structure of the launcher.

\section{Recoil}

With a powder gun, the accelerative force is provided by the high pressure gases generated from the propellant combustion process. Clearly, the recoil force is transmitted at the breech by the propellant gas pressure. The time integral of this force is the recoil impulse and for a powder gun is equal to the momentum imparted to both the projectile and the propellant gases as they exit the muzzle.

In the case of EM, there is neither a breech nor propellant gas. As such the recoil impulse is simply equal to the momentum imparted to the projectile at shot exit, $I_{t}=m_{p} V_{o}$, but where is the recoil force applied? The fact that EM guns must recoil is obvious from first principles and has been measured by mountin $g$ an EM gun in a ballistic pendulum [1]. Witalis [2] postulates that the recoil force is transferred in part at the rails themselves. Marshall and Wood [3] counter that the relativistic effects Witalis claims for rail recoil are simply to small to account for the actual recoil levels. They place the recoil forces at the buswork and power supply. Clearly, the buswork connecting the power circuit to the gun experiences substantial loads as is evidenced by the frequent failure of these components in test. It would be of interest to conduct a careful experiment to define the location of the recoil force. It will be assumed in this paper that the position taken by Marshall and Wood is correct.

To make a comparison with the powder guns, their recoil impulse is estimated as the sum of the projectile momentum and powder gas momentum efflux at the weapon muzzle [4]:

$$
\begin{aligned}
I_{t}= & m_{p} V_{o}+m_{c}\left(R T_{o}\right)^{1 / 2}(2 /(\gamma+1))^{3 / 2} \\
& \left(1+(\gamma+1) m_{c} /\left(12 \gamma m_{p}\right)\right) \\
& {[N \cdot s] }
\end{aligned}
$$




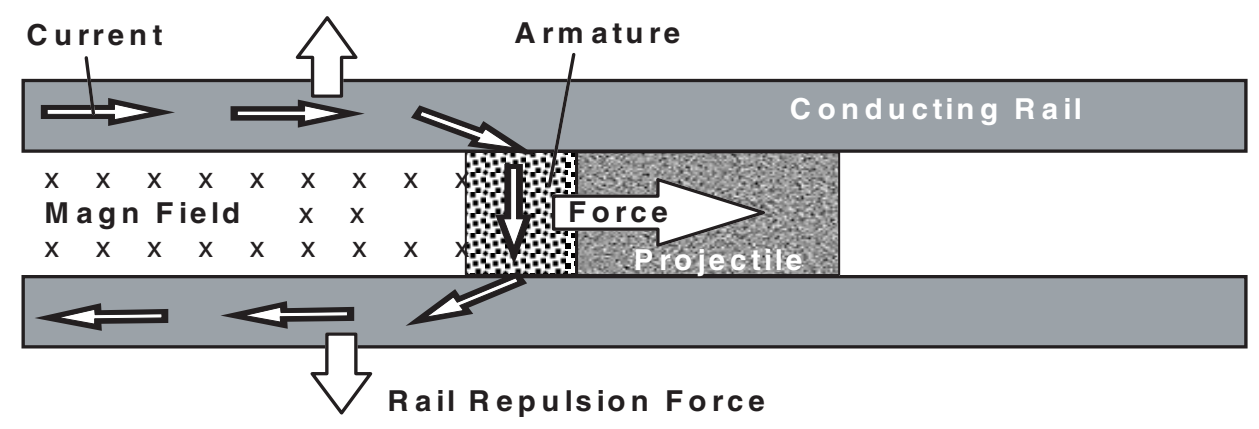

Fig. 1. EM railgun schematic.

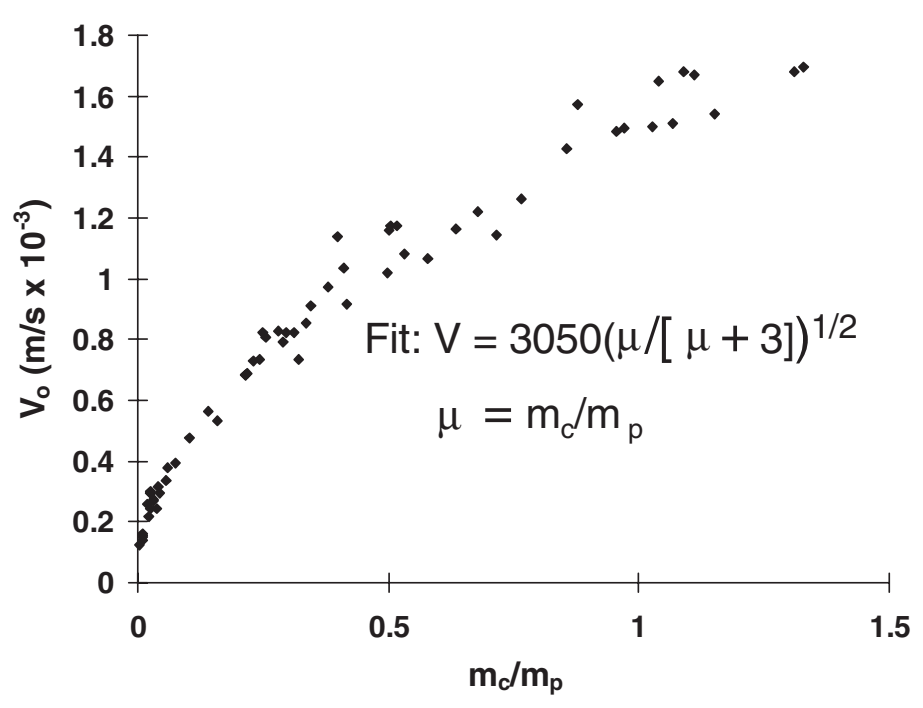

Fig. 2. Velocity vs charge to mass ratio for a wide range of powder guns.

$$
\begin{gathered}
R T_{o}=R T_{a d}-0.26\left(1 / 6+4 m_{p} / 7 m_{c}\right) V_{o}^{2} \\
{\left[m^{2} / s^{2}\right]}
\end{gathered}
$$

where $m_{p}, m_{c}$ are the projectile and charge masses; $V_{o}$ is the projectile muzzle velocity; $R, \gamma$ are the gas constant and ratio of specific heat; and $T_{a d}, T_{o}$ adiabatic flame and average gas temperatures at shot exit. As a simple means to develop the trend in the impulse with velocity, a fit [5] to the correlation presented in Fig. 2 is used.

Using the fit above, Eqs (1) and (2) are rearranged to

$$
\begin{aligned}
& I_{t} / m_{p}= 3050(\mu /[\mu+3])^{1 / 2}+\mu\left(R T_{o}\right)^{1 / 2} \\
&(2 /(\gamma+1))^{3 / 2}(1+(\gamma+1) \\
&\mu /(12 \gamma)) \quad[m / s] \\
& R T_{o}=R T_{a d}-2.4 \times 10^{6}(1 / 6+4 / 7 \mu) \\
&(\mu /[\mu+3]) \quad\left[m^{2} / s^{2}\right]
\end{aligned}
$$

A sample calculation is made on a $20 \mathrm{~mm}$ cannon using WC870 propellant. For this case, $\gamma=1.25, R=$ $365.5 \mathrm{~m}^{2} / \mathrm{s}^{2} \mathrm{~K}$, and $T_{a d}=2577 \mathrm{~K}$. The variation in the impulse ratio for a powder gun is computed and compared to that for an EM gun, $I_{t} / m_{p}=V_{o}$, in Fig. 3 . This ratio could be considered the impulse imparted in firing a constant launch mass. It can be seen that the recoil impulse of the powder and EM are quite similar at low velocities; however, beyond $1800 \mathrm{~m} / \mathrm{s}$ the recoil impulse ratio for the powder gun begins to increase dramatically as the propelling charge mass grows.

Often, comparisons are made by assuming that the gun muzzle energy is constant across the range of launch velocities, Fig. 4. The EM gun behavior simply reflects the fact that the recoil momentum varies as the energy divided by the velocity. For the powder gun, the recoil impulse is similar to that of the EM gun until $1800 \mathrm{~m} / \mathrm{s}$ where it begins to climb. However, for the constant energy case, the increase is less dramatic than 


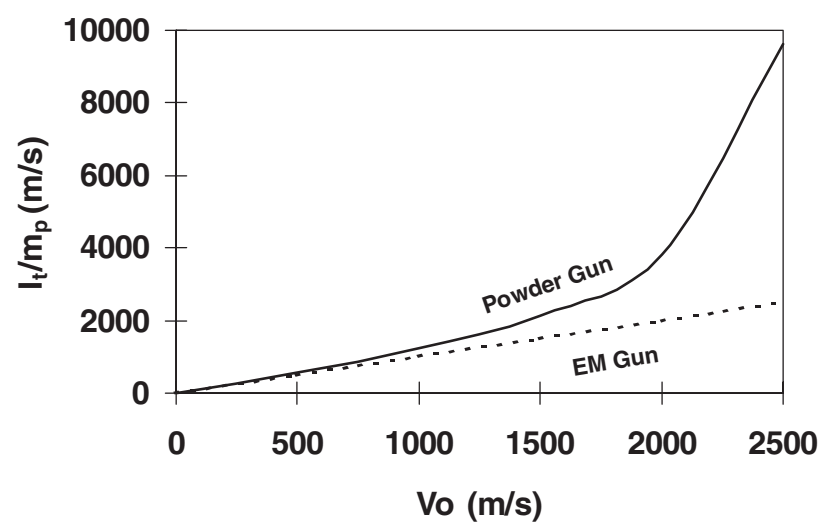

Fig. 3. Impulse ratio vs velocity.

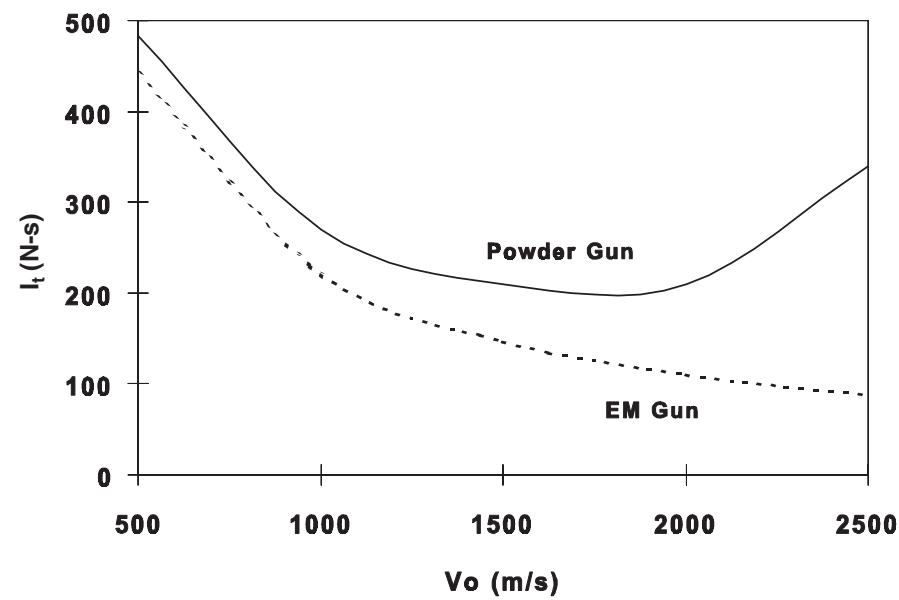

Fig. 4. Constant energy case energy case.

in Fig. 3. For powder guns, there is the potential to decrease the recoil impulse through the use of a muzzle brake.

\section{Muzzle brakes}

Muzzle brakes deflect the exhausting propellant gases laterally thereby recovering axial momentum. The M109 brake, Fig. 5, is a high efficiency device employed on a $155 \mathrm{~mm}$ self-propelled howitzer. Baur and Schmidt [6] measured the recoil attenuation of $20 \mathrm{~mm}$ scale models of M109 and M198 brakes, Fig. 6. The brake momentum efficiency factor is defined

$$
\beta=\left(I_{w o}-I_{w}\right) /\left(I_{w o}-m_{p} V_{o}\right)
$$

The numerator is simply the difference in total recoil impulse with and without the brake in place and the denominator is the total momentum available in the

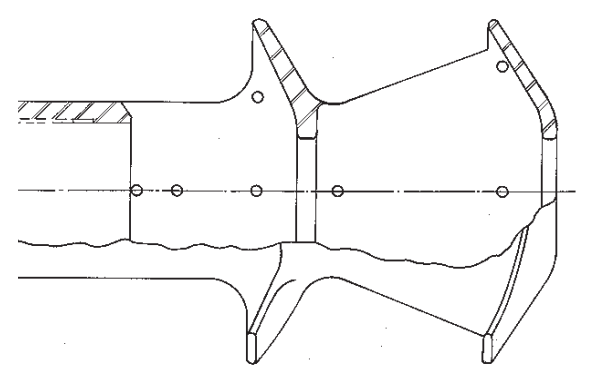

Fig. 5. M109 muzzle brake.

propellant gases. This ratio can exceed $100 \%$ since the gases may be both turned rearward and further expanded. The behavior of $\beta$ in Fig. 6 reflects this. For the $20 \mathrm{~mm}$ cannon as the launch velocity increases, the muzzle pressure also increases. The dimensions of the propellant gas exhaust plume grow with muzzle pressure. The M198 brake is only 1.6 calibers in radius 


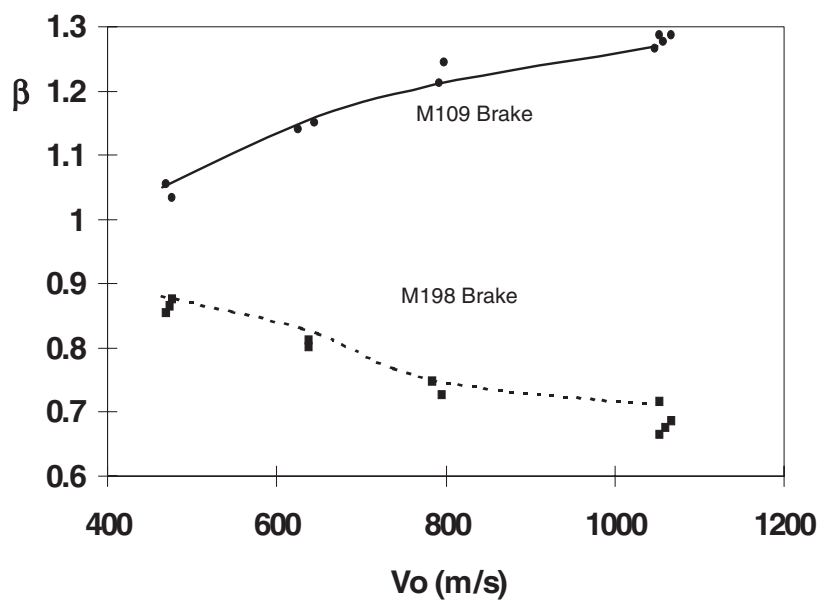

Fig. 6. Muzzle brake efficiencies.

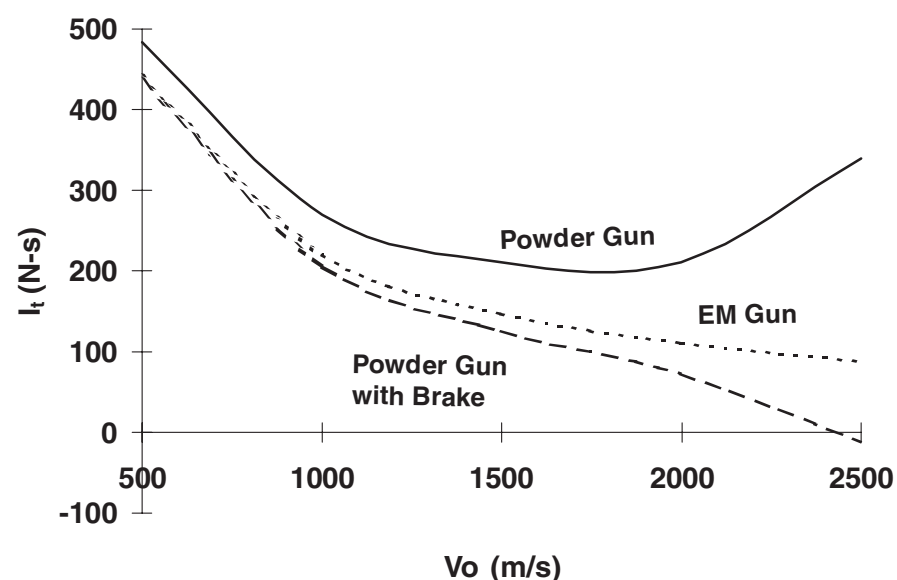

Fig. 7. Constant muzzle energy case with muzzle brake.

and is swept away from the muzzle; thus, its outer edge allows gases to escape as the plume grows. However, the $\mathrm{M} 109$ brake has a radius of 2.0 calibers and is swept toward the muzzle. This baffle captures more of the plume as pressure increases and the brake efficiency grows. Eventually, the supersonic core of the plume should fully envelop both brake surfaces and variation with velocity (pressure) should level out. These data may be used to estimate the impulse imparted to the cannon.

By fitting the M109 data in Fig. 6 and extrapolating to velocities up to $2500 \mathrm{~m} / \mathrm{s}$, the impulse of a powder gun with a high efficiency brake may be compared to the impulse of an EM gun over a similar range, Fig. 7. Since the efficiency of the M109 brake is greater than one, the total impulse imparted to the powder gun with the brake installed is lower than the impulse imparted to the EM gun, i.e., the momentum imparted to the projectile. There are drawbacks to employing a muzzle brake. The M109 brake is quite massive and affects the gun dynamics and the design of the elevating and traversing mechanisms. Also, the employment of a muzzle brake greatly increases the muzzle blast overpressure behind the weapon. This may not be a problem with a vehicle having a closed hatch, but infantry moving with an armored fighting vehicle could be injured.

\section{Conclusions}

The recoil characteristics of an electromagnetic railgun are considered and compared with those of a powder gun. The EM gun is shown to have less recoil 
impulse than does a powder gun, particularly at high velocities. However, the installation of a muzzle brake can alter this comparison to the point where the recoil imparted to a powder gun equipped with a high efficiency brake is lower than that of an EM gun.

\section{References}

[1] A. Zielinski, private communication of results presented at the 11th EM Launcher Association Meeting, Sep. 1998, pp. 93.
[2] E. Witalis, Origin, Location, Magnitude, and Consequences of Recoil in Plasma Armature Railgun, IEEE Proc. A 142(3) (1995).

[3] R. Marshall and L. Woods, private communication of draft paper responding to Witalis, 1996.

[4] C. Lang, Muzzle Devices, AMCP 706-251, AMC, Alexandria, VA, May, pp. 68.

[5] E. Schmidt, A Ballistic Evaluation of Electromagnetic Launch and Flight, Proceedings of 17th Int'l Symposium on Ballistics, NDIA, Arlington, VA, May, pp. 98.

[6] E. Baur and E. Schmidt, Relationship between Efficiency and Blast from Gas Dynamic Recoil Brakes, AIAA Paper 85-1718, July, pp. 85. 

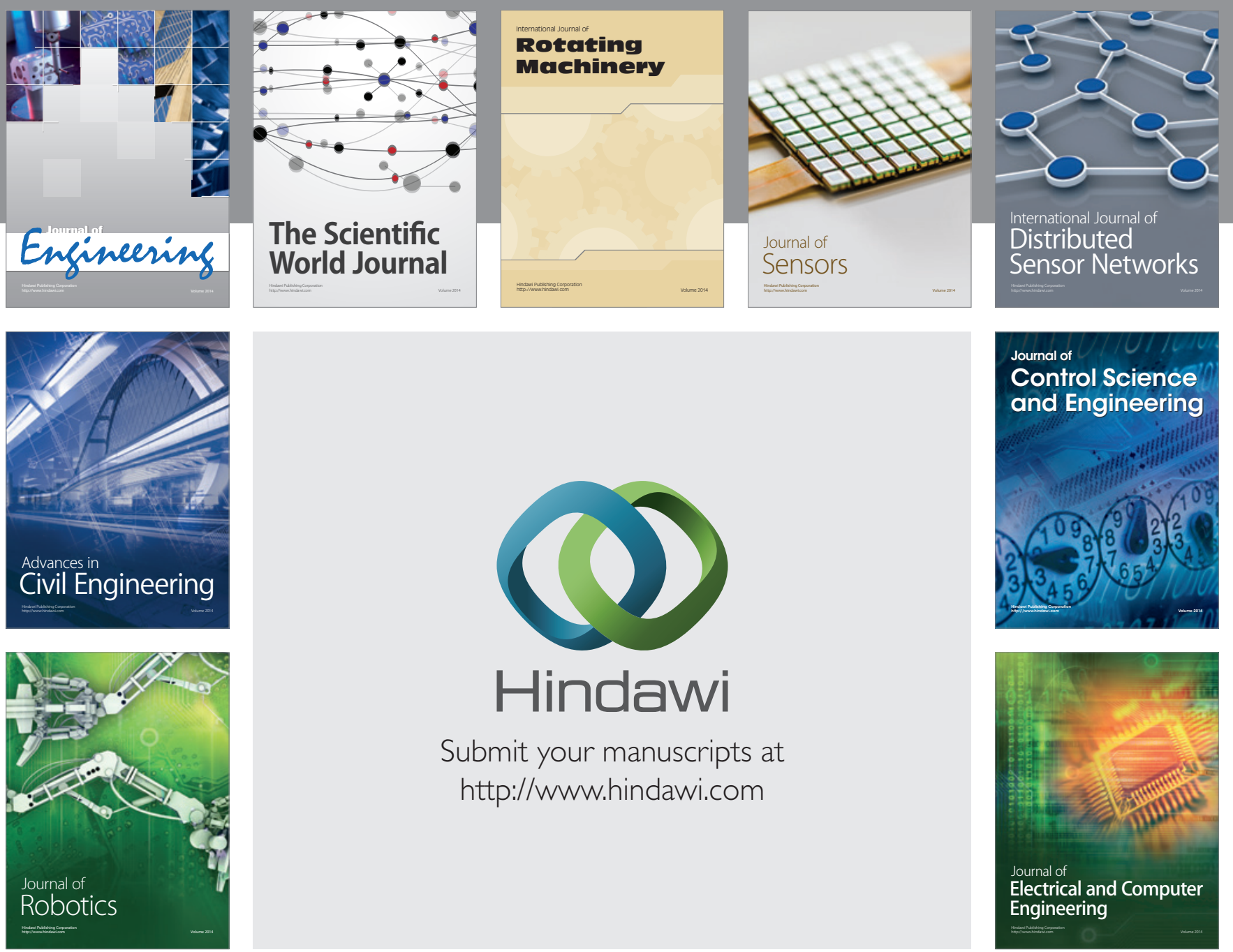

Submit your manuscripts at

http://www.hindawi.com
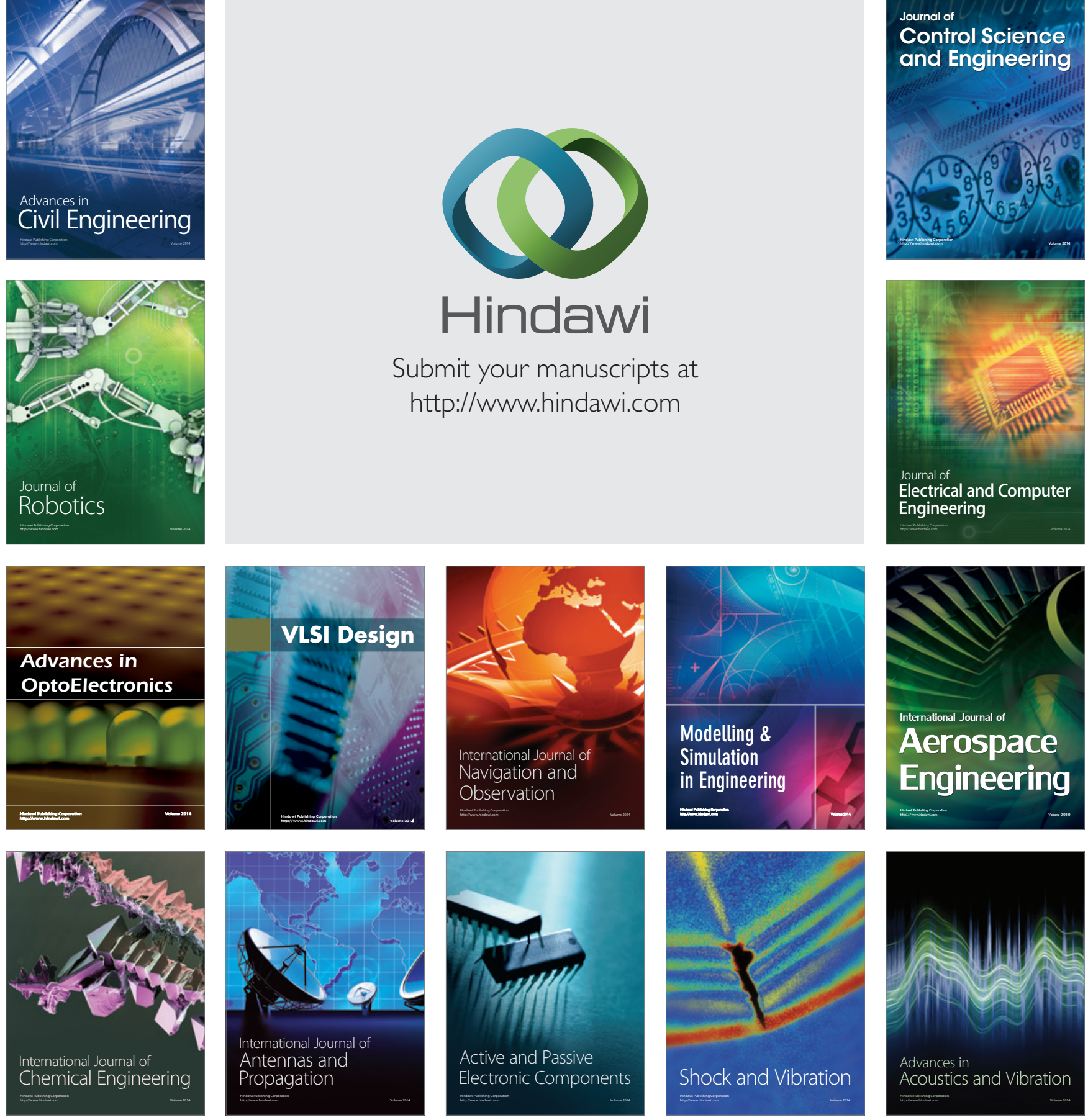